\title{
Linking carbonate sand fabric and mechanical anisotropy from hollow cylinder tests: motivation and application
}

\author{
Ming Fook Lim ${ }^{1}$, J. Antonio H. Carraro, Ph.D. ${ }^{2}$, and \\ Susan Gourvenec, Ph.D. ${ }^{3}$
}

\begin{abstract}
${ }^{1} \mathrm{PhD}$ Student, Centre for Offshore Foundation Systems, The University of Western Australia, 35 Stirling Highway, Perth WA 6009; e-mail: mingfook.lim@research.uwa.edu.au

${ }^{2}$ Associate Professor, Centre for Offshore Foundation Systems, The University of Western Australia, 35 Stirling Highway, Perth WA 6009; e-mail: antonio.carraro@uwa.edu.au

${ }^{3}$ Professor, Centre for Offshore Foundation Systems, The University of Western Australia, 35 Stirling Highway, Perth WA 6009; e-mail: susan.gourvenec@uwa.edu.au
\end{abstract}

\begin{abstract}
In addition to density and stress, fabric is also a key state variable strongly affecting soil behavior. While fabric influence on mechanical behavior of soils has been investigated experimentally, the available database is limited in terms of boundary conditions and soil types tested. Offshore carbonate sediments are of special interest for offshore geotechnical analyses due to their prevalence in tropical waters and unique mechanical behavior that stems from their mostly biogenic origin. A key gap in the availability of experimental data on soil fabric relates to the anisotropy of offshore carbonate sediments. In practice, anisotropy studies (whether rigorously correlated to fabric or not) are typically carried out experimentally for simple boundary conditions such as idealized plane strain and axisymmetric states. In real geotechnical applications, stress paths subjected to soil elements in the field are far more complex, often involving the combined variations of both the orientation and magnitude of all three principal stresses. This paper presents a new multiscale approach to assess soil fabric at the micro-scale level and relate it to the macromechanical response observed for generalized loading conditions. A new sampling method is illustrated that enables preservation and evaluation of the fabric of offshore sediments specimens following generalized stress disturbances imparted by a hollow cylinder apparatus. The link between fabric evolution and the observed stress-strain behavior of sand is discussed along with preliminary results. The approach is part of a broad framework that will be used to systematically study the evolution of soil fabric and anisotropy and their relationship to multi-directional loading scenarios.
\end{abstract}




\section{INTRODUCTION}

Soil fabric is the arrangement of particles, particle groups and associated voids within a soil mass (Mitchell and Soga 2005). Whereas the role of soil fabric is typically ignored in conventional geotechnical design and analysis, its impact on soil behavior has been demonstrated by element tests on similar specimens prepared with different fabric by different reconstitution methods (Chaudhary et al. 2002; Murthy et al. 2007; Tatsuoka et al. 1986; Vaid et al. 1999).

Fundamental research into the role of soil fabric has been carried out using both imaging of preserved soil fabric from element tests (Fonseca et al. 2013; Frost and Jang 2000; Oda 1972) and discrete element method (DEM) simulations (Ai et al. 2014; Tong et al. 2014). However, major gaps still exist on the quantification of soil fabric from element tests for certain soils. DEM may be a crucial tool to better understand and model particulate soil behavior, but models must always be validated by relevant, quality experimental data, which is sparse for generalized loading scenarios. Even though approaches are available to preserve and quantify soil fabric in triaxial tests (Fonseca et al. 2013; Jang et al. 1999), further attempts have not been made to include other element tests that are capable of applying more general boundary conditions. The acquisition of soil fabric data and its evolution from element tests whose boundary conditions are more complex than axisymmetric ones is important because most real world applications result in non-proportional loading conditions, leading to changes in both orientation and magnitude of principal stresses. Such changes are conveniently represented by the angle of major principal stress orientation with respect to vertical axis $(\alpha)$ and the intermediate principal stress ratio (b). Examples of real world offshore applications where relevant variations in magnitude and orientation of principal stresses take place are schematically shown in Figure 1.

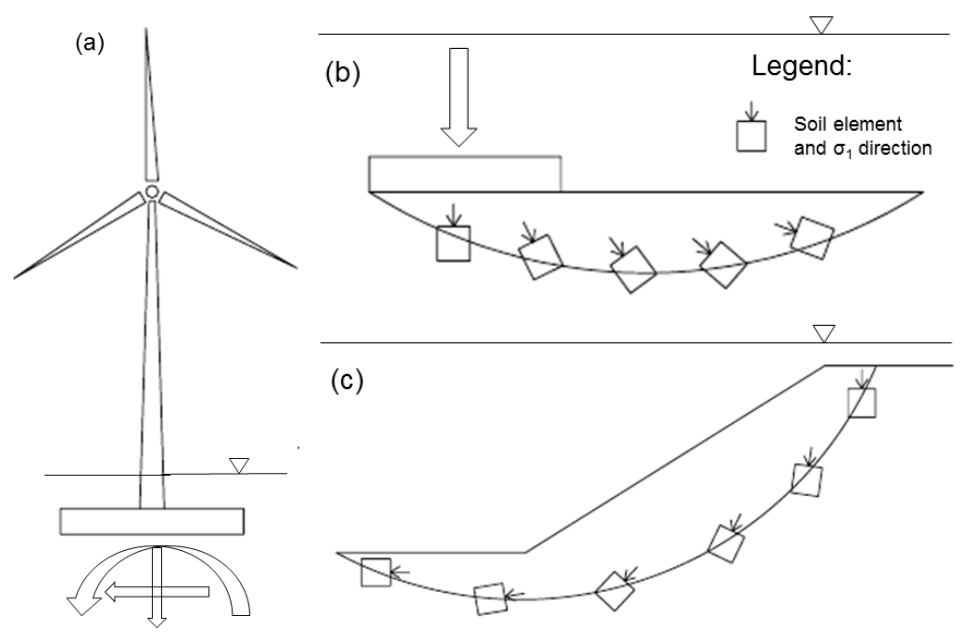

Figure 1. Offshore examples of (a) multi-directional loading under wind turbine and major principal stress rotation under (b) shallow foundation and (c) submarine slope. 
With the complex stress state generated under a simple shallow foundation, let alone in more complex problems such as multi-directional loading of foundations and/or anchors supporting offshore infrastructure subjected to installation, operational and environmental loads, it is crucial that the role of soil fabric be evaluated for soil that is subjected to non-proportional loading. Even though advanced experimental techniques exist now that allow systematic reconstitution of hollow cylinder specimens for sandy deposits subjected to deposition under water (Tastan and Carraro 2013), high-quality experimental investigations into the fabric evolution of carbonate sediments under non-proportional loading are non-existent. Carbonate sediments are particularly relevant to offshore geotechnics due to the prevalence of carbonate seabeds in tropical waters and their different mechanical behavior compared to the behavior of terrigenous soils (McClelland 1988; Milliman 1974). Thus, the development of a methodology that would allow rigorous assessment of soil fabric evolution upon nonproportional loading scenarios is in order for both carbonate and non-carbonate soils. This would help extend previous rigorous research ( $\mathrm{Li}$ and $\mathrm{Yu} 2014$ ) on the role of fabric on the mechanical behavior of soils.

\section{METHODOLOGY}

The methodology for investigating the relationship between soil fabric and anisotropy for offshore carbonate sands is divided into three stages, as described next.

\section{Hollow Cylinder Testing}

The first stage relates to the testing procedure used for applying non-proportional loading. In order to explore more realistic and relevant stress paths, the hollow cylinder apparatus is used due to its greater versatility in applying loads that are more three-dimensional in nature and therefore more representative of real world applications. This is achieved by subjecting hollow cylindrical soil specimens to different combinations of external loads (Figure 2). By varying the inner cell pressure $\left(\mathrm{P}_{\mathrm{i}}\right)$, outer cell pressure $\left(\mathrm{P}_{\mathrm{o}}\right)$, axial load $(\mathrm{V})$ and torque $(\mathrm{T})$, the stress state in a soil element from the specimen wall can be induced as shown in Figure 2.

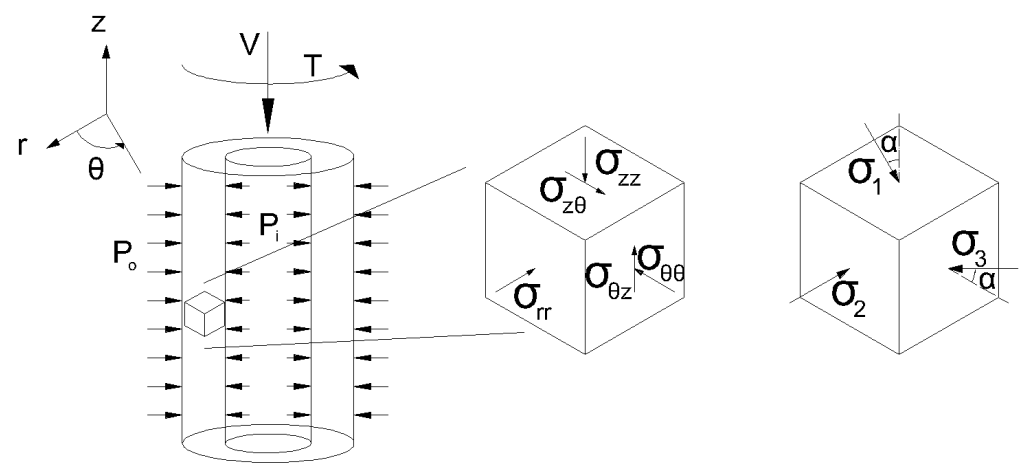

Figure 2. Hollow cylinder specimen and stress components induced to element within specimen wall (modified after Hight et al. 1983). 
In hollow cylinder testing the orientation of major and minor principal stresses can be rotated about an axis in a continuous and controlled manner, unlike the stress state in a conventional triaxial test. The intermediate principal stress can also be systematically controlled during the tests. The ability to control $\alpha$ and $b$ allows a systematic study of soil anisotropy due to controlled changes in the orientation of major principal stress and intermediate principal stress magnitude (Hight et al. 1983).

\section{Preservation of Soil Fabric}

Hollow cylinder testing allows soil anisotropy to be studied systematically from analysis of stress-strain data but the process does not yield parameters about the fabric tensor directly. To obtain quantitative information about specimen fabric at any test stage, an epoxy impregnation technique is used. This was achieved by modifying existing methodologies for preservation of triaxial specimens (Fonseca et al. 2013; Jang et al. 1999). Figure 3a shows one step of the new epoxy-impregnation method used. Gelatin solution is first introduced through the base pedestal to solidify the lower third of the specimen and prevent permanent clogging of the porous element (Fig. 3b) by epoxy. Epoxy is introduced later through the additional line shown in Fig. 3a. The gelatin and epoxy used in this study were similar to those used by Carraro and Prezzi (2008) and Jang et al. (1999), respectively. A detailed description of the new epoxy-impregnation method developed in this research is out of the scope of the present paper but will be presented in a future publication.
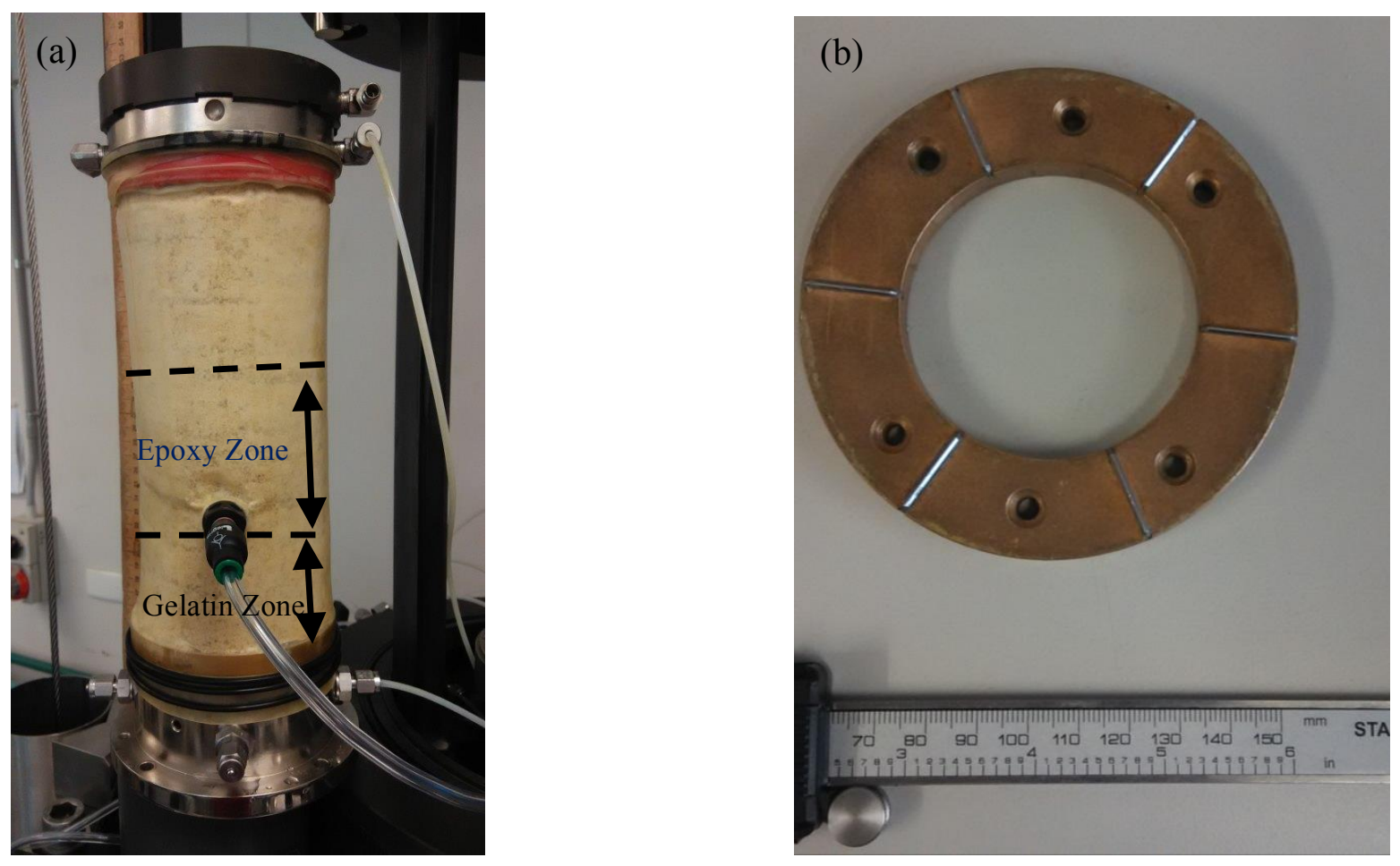
Figure 3. (a) Hollow cylinder specimen (before test) showing the epoxy impregnation line, (b) Porous element from the base pedestal.

\section{Micro-Computed Tomography}

After epoxy impregnation, a sub-sample from the epoxy zone with width equal to approximately $30 \mathrm{~mm}$ (Figure $4 \mathrm{a}$ ) is cut with a diamond saw. This sub-sample is then placed within a micro-computed tomography device (Fig. 4b) to generate scanned images of the internal soil structure for quantitative analysis.
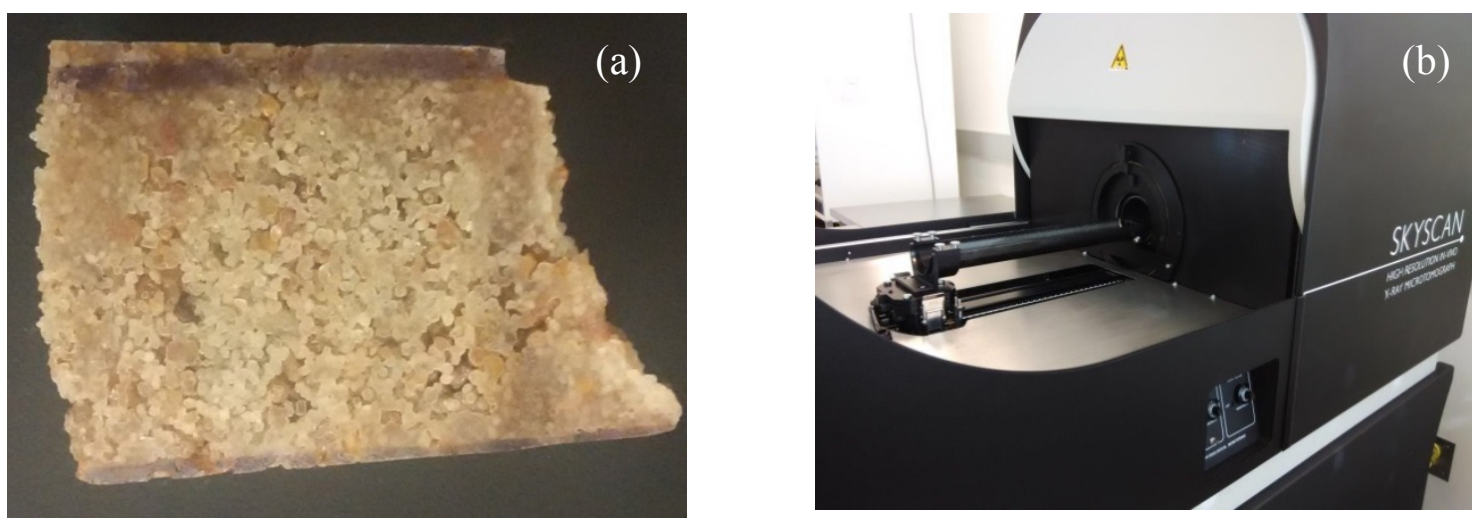

Figure 4. (a) Epoxy-impregnated sub-sample from a hollow cylinder specimen; (b) micro computed tomography apparatus.

Soil fabric quantification may be carried out using various approaches. Detailed descriptions of the approaches available for soil fabric quantification are presented elsewhere (Fonseca et al. 2013; Oda 1972; O'Sullivan 2011). Soil fabric may be assessed in a relatively simple way through quantification of the preferred orientation of major particle axis (Oda 1972). Alternatively, contact normals between randomly selected normal particle contacts for randomly selected 2D slices of a sample can also be assessed (Oda 1972). These methods may be applied to any orientation, including vertical and horizontal slices, for example. In another approach, rose diagrams showing the intensity of contact normals can be produced to assess whether contact normals feature any preferential direction. With a rose diagram, the approximate distribution of contact normals may be expressed as (Rothenburg and Bathurst 1992)

$\mathrm{E}(\theta)=\frac{1}{2 \pi} \times\left[1+\beta \cos \left(\theta-\theta_{0}\right)\right]$

Where: $\mathrm{E}(\theta)$ is the normalized contact normal distribution, $\beta$ is a measure of contact normal anisotropy, and $\theta_{0}$ represents the orientation of the major principal axis of contact normals. Parameters such as $\beta$ and $\theta_{0}$ (or others that might relate to major particle axis orientation, for example), can be quantified from micro-computed tomography analysis and then systematically compared to the observed stress-strain response ( $\mathrm{Li}$ and $\mathrm{Yu}$ 2014). 
Thus, measured changes in quantifiable soil fabric parameters could be directly related to controlled disturbances imparted during hollow cylinder tests as well. The evolution of contact normals or major particle axis orientations, for example, may be tracked for different stages of the stress path imposed in hollow cylinder tests. Characterization of soil fabric at various stages of a stress path will naturally involve repeating tests on similar specimens and stopping the test at the desired stage for further soil fabric quantification.

\section{PRELIMINARY RESULTS AND DISCUSSION}

The epoxy impregnation and use of micro-computed tomography described above was initially applied to a clean sand specimen. This was done to generate preliminary results and assess the feasibility of the proposed approach of evaluating soil fabric evolution due to controlled general loading disturbances in hollow cylinder testing.

\section{Imaging and Soil Fabric}

Figure 4a shows an example of an epoxy-impregnated sub-sample from a hollow cylinder specimen obtained with the methodology described in this paper. This suggests the proposed epoxy-impregnated method can be systematically used to obtain samples for micro-computed tomography. The maximum axial strain observed during epoxy impregnation of a clean sand specimen was $0.11 \%, 0.01 \%$ and $0.02 \%$ for backpressure reduction, drying and gelatin impregnation stages, respectively.

During the subsequent micro-computed tomography stage, such small samples can be easily scanned and the data analyzed so as to produce the desired images for further fabric quantification. To illustrate this, Figure 5 shows examples of 2D sections of the internal fabric of an offshore carbonate sand identical to that tested by Carraro and Bortolotto (2015). These 2D sections were reconstructed from the micro-computed tomography data. This sample was simply deposited under water and not subjected to further loading.

From the fabric data obtained from micro-computed tomography, 2D images were reconstructed and two randomly selected slices were chosen, a horizontal and a vertical one. In spite of the inherent complexity associated with the fabric of offshore carbonate sediments, a few qualitative features can be promptly identified from Fig. 5. First, many particles are hollow and/or present intra-particle voids due to their biogenic origin. Such features could have very important mechanical implications if particles break and/or are crushed. This could decrease the void ratio of the sample by unpredictable amounts possibly leading to either specimen deformation in the laboratory or settlement of the deposit in the field. Secondly, it would be expected that water deposited sands would show cross-anisotropic fabric (i.e. isotropic in horizontal slice and anisotropic in vertical slice) due to gravitational effect on particle sedimentation. To assess this, frequency distributions of maximum particle 
dimension orientations $(\varphi)$ for the 2D sections were quantified (Oda 1972) and are presented in Figure 6, confirming the cross-anisotropic fabric hypothesis.

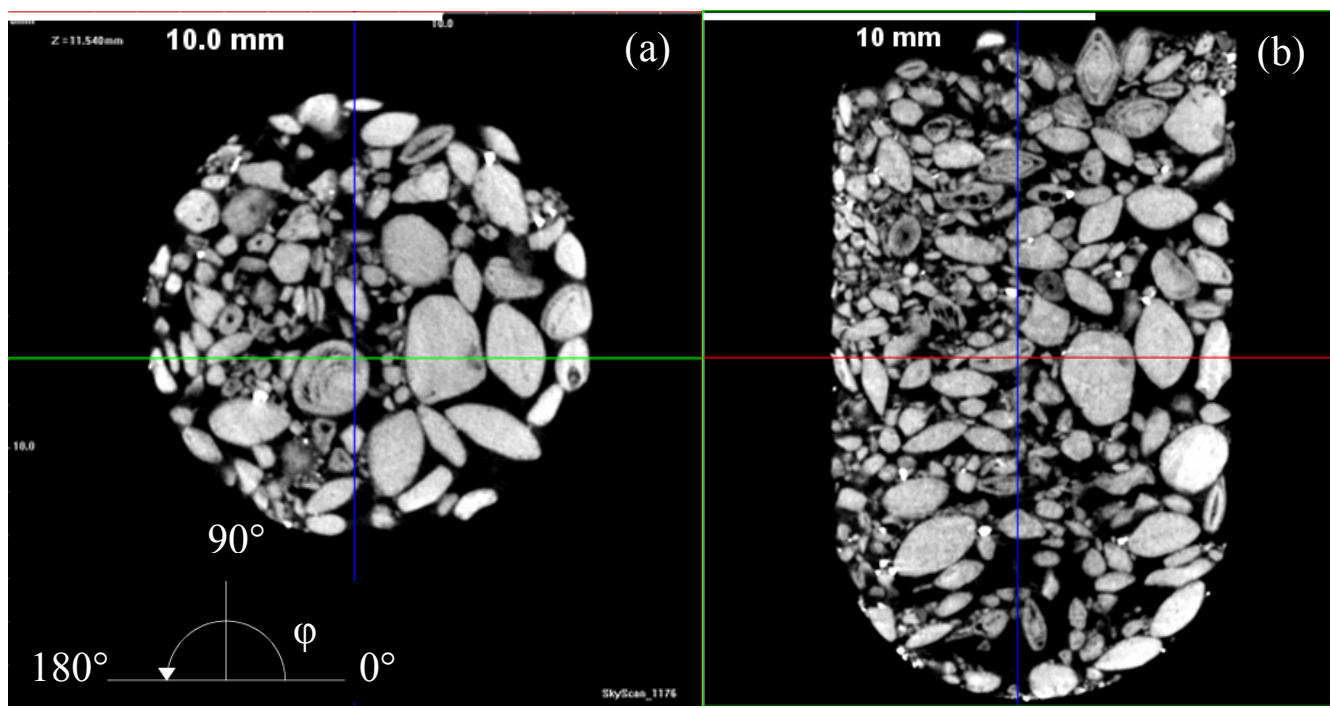

Figure 5. Offshore carbonate sand fabric from micro-computed tomography: (a) horizontal and (b) vertical slices. The angle $(\varphi)$ showed in Figure 5(a) is used for quantification of maximum particle axis orientation in both slices.
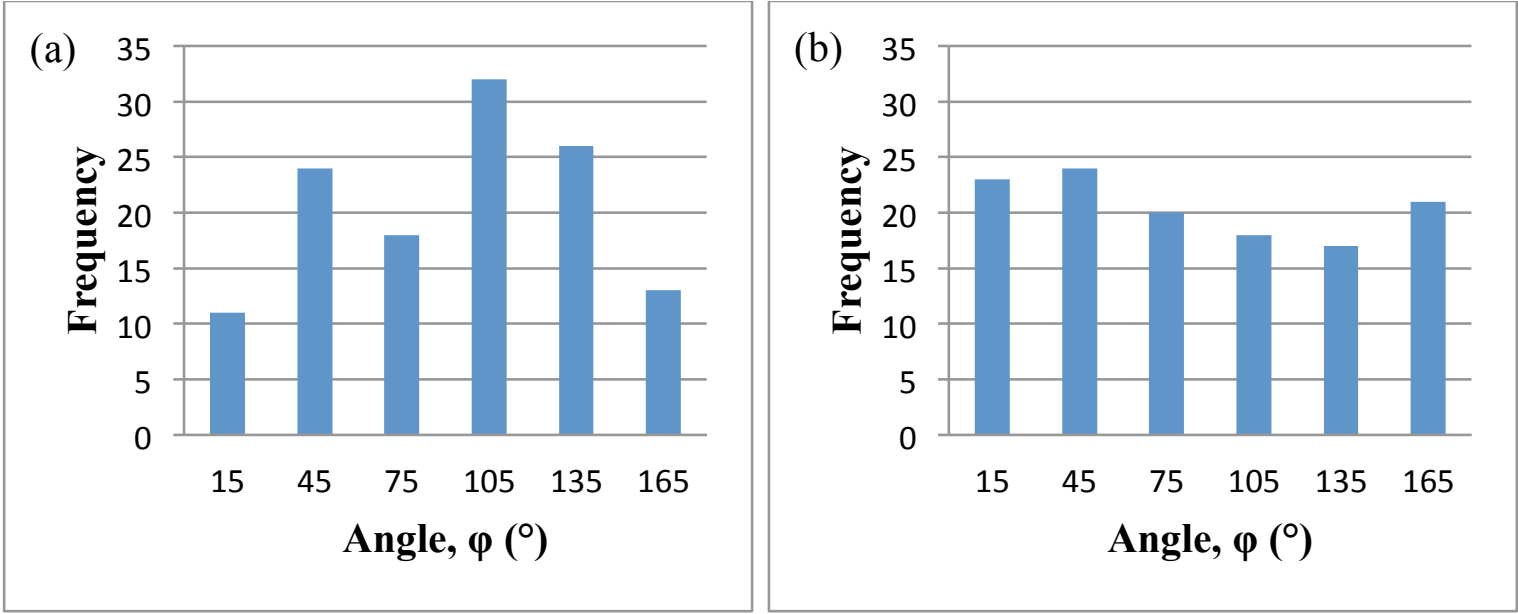

Figure 6. Frequency distribution of major particle dimension orientation for (a) horizontal and (b) vertical slices shown in Fig. 5.

\section{Soil Fabric Evolution}

Figure 7 shows preliminary results of a hollow cylinder test on a clean sand sample. The specimen was (1) backpressure saturated; (2) anisotropically consolidated; (3) subjected to 
undrained principal stress rotation with $\alpha$ and $b$ increasing to 75 degrees and 0.5 , respectively; (4) maintained at constant stress and impregnated with epoxy resin for the subsequent micro-computed tomography analysis. An idealized response for an additional stage to be carried out on a similar hollow cylinder specimen is also shown in Fig. 7 during which the specimen would undergo additional undrained increases in the octahedral deviator stress (q) until large strains are mobilized while keeping both $\alpha$ and b constant. For comparison purposes, similar idealized responses for two additional tests carried out following different levels of principal stress rotations (30 and 55 degrees) are also shown in Fig. 7. Measured stress states anywhere along the stress-strain responses (Fig. 7) would then be correlated to the corresponding, quantified soil fabric evolution from micro-computed tomography analysis.

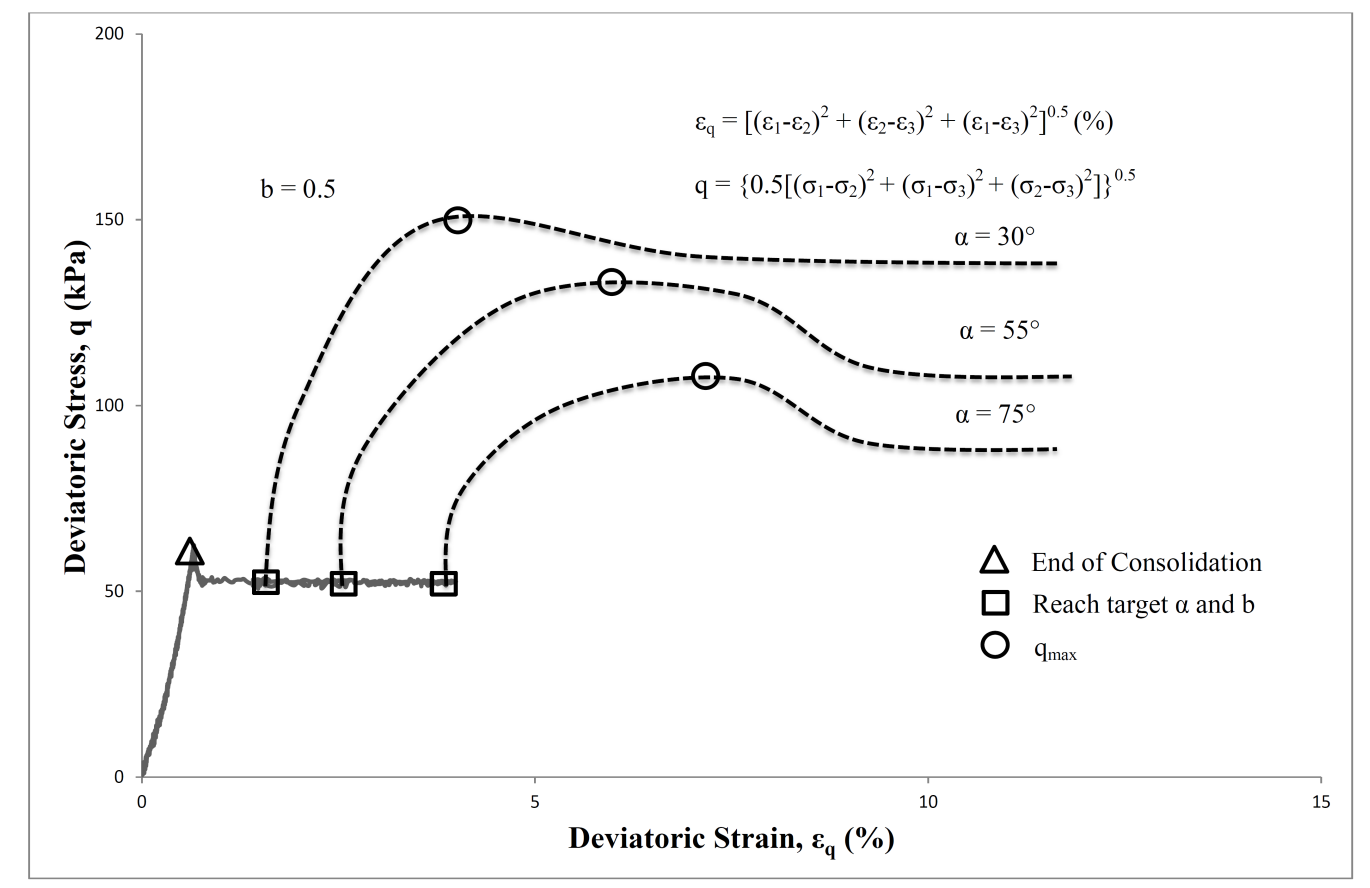

Figure 7. Hollow cylinder tests on clean sand specimens subjected to anisotropic consolidation and controlled undrained changes in $\alpha$ and $b$. Idealized responses for other stages and/or different combinations of $\alpha$ and $b$ shown in dashed lines.

Stress-strain curves from hollow cylinder tests can be related to quantified soil fabric from imaging of preserved samples. Figure 8 shows an idealized curve of evolution of the anisotropy of contact normals $(\beta)$ at different testing stages. This information can be subsequently used to derive correlations between evolving soil fabric and stress-strain behavior from hollow cylinder tests. By running a series of tests on similar soil specimens with different combinations of $\alpha$ and $b$ and ending tests at different stages (e.g. end of consolidation, after applying target $\alpha$ and $b$, etc.), soil fabric evolution can be quantified as schematically shown in Figure 8. 


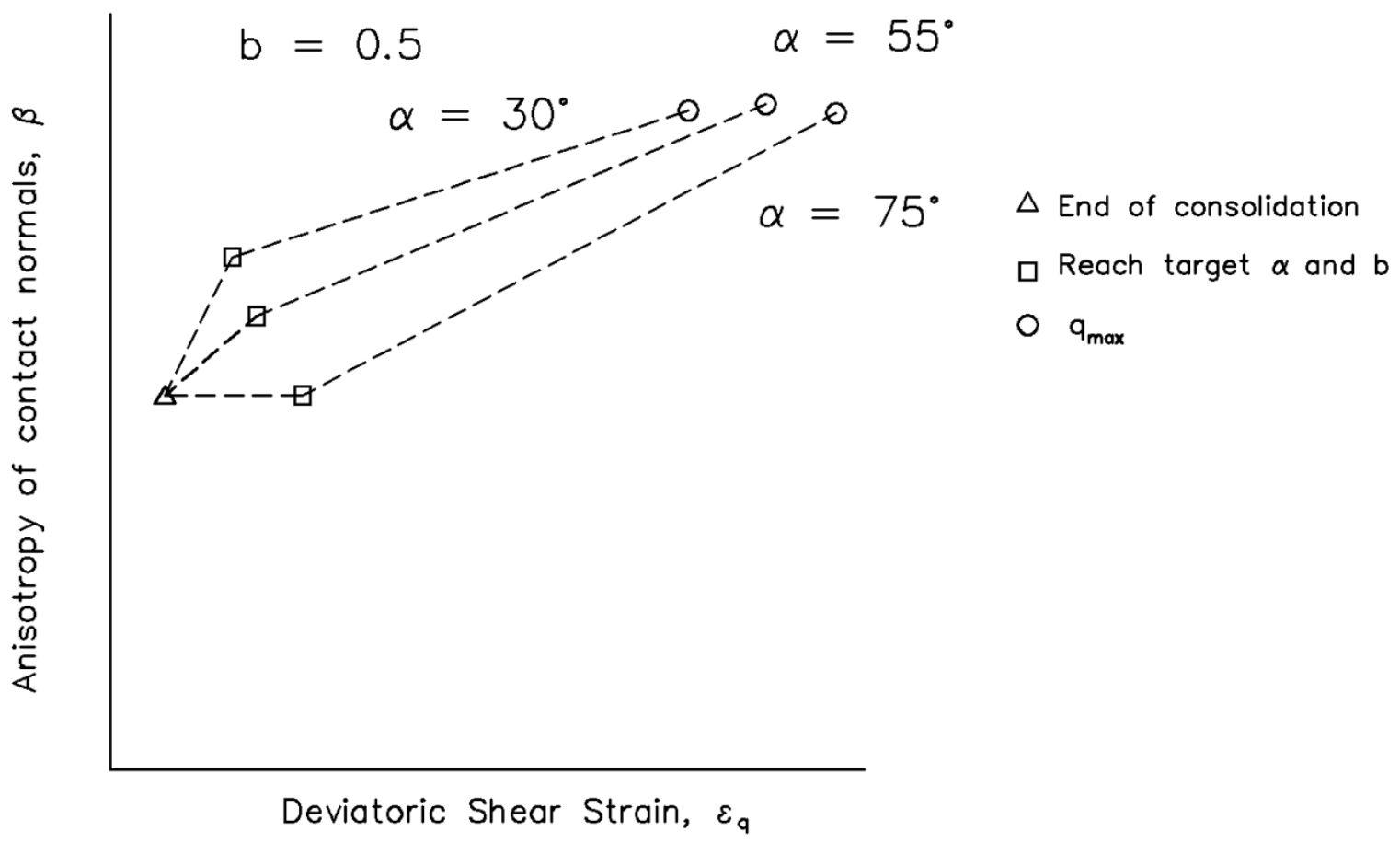

Figure 8. Evolution of contact normal anisotropy $(\beta)$ with deviatoric shear strain.

\section{CONCLUSIONS}

A framework is presented to link soil fabric evolution and stress-strain behavior from hollow cylinder tests. The framework includes (1) hollow cylinder testing to obtain stress-strain behavior for different combinations of $\alpha$ and $b$, (2) a new epoxy impregnation method to preserve soil fabric in hollow cylinder tests, (3) quantification of soil fabric using microcomputed tomography, and (4) comparison of stress-strain behavior and soil fabric evolution.

Steps (2) and (3) of the proposed framework were employed in this preliminary study to apply epoxy impregnation to a hollow cylinder specimen for further fabric analysis. 2D sections of an offshore carbonate sand fabric were reconstructed from micro-computed tomography data and analysed to show cross anisotropy.

\section{REFERENCES}

Ai, J., Langston, P. A., and Yu, H.-S. (2014). "Discrete element modelling of material noncoaxiality in simple shear flows." International Journal for Numerical and Analytical Methods in Geomechanics, 38(6), 615-635.

Carraro, J.A.H. and Bortolotto, M.S. (2015) "Stiffness degradation and damping of carbonate and silica sands." Frontiers in Offshore Geotechnics III. London, UK, CRC Press/Balkema, 1179-1183.

Carraro, J.A.H. and Prezzi, M. (2008). "A New Slurry-Based Method of Preparation of Specimens of Sand Containing Fines.” Geotechnical Testing Journal, 31(1), 1-11. 
Chaudhary, S. K., Kuwano, J., Hashimoto, S., Hayano, Y., and Nakamura, Y. (2002). "Effects of Initial Fabric and Shearing Direction on Cyclic Deformation Characteristics of Sand." Soils and Foundations, 42(1), 147-157.

Fonseca, J., O'Sullivan, C., Coop, M. R., and Lee, P. D. (2013). "Quantifying the evolution of soil fabric during shearing using directional parameters." Géotechnique, 63(6), 487-499.

Frost, J. D., and Jang, D.-J. (2000). "Evolution of sand microstructure during shear." Journal of Geotechnical and Geoenvironmental Engineering, 126(2), 116-130.

Hight, D. W., Symes, M. J., and Gens, A. (1983). "The development of a new hollow cylinder apparatus for investigating the effects of principal stress rotation in soils." Géotechnique, 33(4), 355-383.

Jang, D.-J., Frost, J., and Park, J.-Y. (1999). "Preparation of Epoxy Impregnated Sand Coupons for Image Analysis." Geotechnical Testing Journal, 22(2), 153.

Li, X., and Yu, H.-S. (2014). "Fabric, force and strength anisotropies in granular materials: a micromechanical insight." Acta Mechanica, 225(8), 2345-2362.

McClelland, B. (1988). "Calcareous sediments: An engineering enigma." A.A. Balkema, Perth, 777-786.

Milliman, J. D. (1974). Recent Sedimentary Carbonates: Part 1 Marine Carbonates. Springer, Berlin.

Mitchell, J. K., and Soga, K. (2005). Fundamentals of soil behavior. Series in soil engineering, John Wiley \& Sons, Inc., Hoboken, New Jersey.

Murthy, T. G., Loukidis, D., Carraro, J. a. H., Prezzi, M., and Salgado, R. (2007). "Undrained monotonic response of clean and silty sands." Géotechnique, 57(3), 273-288.

Oda, M. (1972). "Initial Fabrics and Their Relations to Mechanical Properties of Granular Material." Soils and Foundations, 12(1), 17-36.

O’Sullivan, C. (2011). Particulate discrete element modelling: A geomechanical perspective. Spon Press/Taylor \& Francis, London; New York.

Rothenburg, L., and Bathurst, R. J. (1992). "Micromechanical features of granular assemblies with planar elliptical particles." Géotechnique, 42(1), 79-95.

Tastan, E. O., and Carraro, J. A. H. (2013). "A New Slurry-Based Method of Preparation of Hollow Cylinder Specimens of Clean and Silty Sands." Geotechnical Testing Journal, 36(6), 20130056.

Tatsuoka, F., Ochi, K., Fujii, S., and Okamoto, M. (1986). "Cyclic Undrained Triaxial and Torsional Shear Strength of Sands for Different Sample Preparation Methods." Soils and Foundations, 26(3), 23-41.

Tong, Z., Fu, P., Dafalias, Y. F., and Yao, Y. (2014). "Discrete element method analysis of non-coaxial flow under rotational shear." International Journal for Numerical and Analytical Methods in Geomechanics, 38(14), 1519-1540.

Vaid, Y. P., Sivathayalan, S., and Stedman, D. (1999). "Influence of SpecimenReconstituting Method on the Undrained Response of Sand." Geotechnical Testing Journal, 22(3), 187-195. 